\title{
Carbon outclasses wood in racket paddles: Ratings of expert and intermediate tennis players
}

\author{
L. S. OVERNEY ${ }^{1,2}$, V. MICHAUD ${ }^{3}$, C. FISCHER ${ }^{3}$, J. HEUBI $^{1}$, L. VELDHUIS ${ }^{3}$, O. BLANKE ${ }^{1}$, \\ M. H. HERZOG ${ }^{2}$, \& J.-A. MÅNSON ${ }^{3}$ \\ ${ }^{1}$ Laboratory of Cognitive Neuroscience, Ecole Polytechnique Fédérale de Lausanne (EPFL), 1015 Lausanne, Switzerland, \\ ${ }^{2}$ Laboratory of Psychophysics, Ecole Polytechnique Fédérale de Lausanne (EPFL),1015 Lausanne, Switzerland and \\ ${ }^{3}$ Laboratory of Composite and Polymer Technology (LTC), Ecole Polytechnique Fédérale de Lausanne (EPFL),1015 \\ Lausanne, Switzerland
}

(Accepted 4 August 2010)

\begin{abstract}
Wooden racket paddles were modified with rubber and carbon fibre laminates and their differences tested in terms of flexural, damping, and coefficient of restitution properties. Four rackets types were designed: a wood reference, wood with rubber, carbon fibre $0^{\circ}$, and carbon fibre $90^{\circ}$. Seven expert and eight intermediate tennis players tested the rackets. To determine which of the four rackets suited the players best, we asked the players to compare the rackets two by two. After each pair tested, participants had to fill out a 4-item questionnaire in which different aspects of the rackets' performance were judged. The most preferred racket was the $0^{\circ}$ carbon fibre racket, followed by the $90^{\circ}$ carbon fibre racket, the wood racket and, finally, the 1 - $\mathrm{mm}$ rubber racket. Thus, rackets with the highest stiffness, least damping, and highest coefficient of restitution were the most preferred. Interestingly, although experts and intermediate players overall judged the rackets in very similar ways according to force, vibration, and control, they were sensitive to quite different physical characteristics of the rackets.
\end{abstract}

Keywords: Racket, subjective judgement, mechanical properties, vibration

\section{Introduction}

High-level performance in sport requires a combination of precise equipment and highly trained motor and perceptual skills. In a recent study, we tested how advanced and expert skiers judged pairs of skis with different materials (Fischer et al., 2007). We showed that the skis with a low flexural and a high torsional rigidity were highly rated when it came to the general impression of the ski. More specifically, expert skiers were particularly sensitive to the torsion criterion at the front of the skis. These results suggest that such highly rated elements should be taken into consideration for future ski designs.

Apart from Fischer et al. (2007), few researchers have investigated the complex combination of the athlete's perception depending on the mechanical and dynamic properties of the sport equipment. Some studies have examined these issues in golf (Roberts, Jones, Harwood, Mitchell, \& Rothberg, 2001; Roberts, Jones, Mansfield, \& Rothberg, 2005a, $2005 \mathrm{~b}$ ) and showed correlations between the feel of a shot and the vibration and sound of a club. Most of the other studies have either focused on the sweet spot of an implement (Carello, Thuot, Anderson, \& Turvey, 1999; Cross, 1998; Haake, Carré, \& Goodwill, 2003) or on the biomechanics (Cross, 1998; Stroede, Noble, \& Walker, 1999) and have therefore dealt more specifically with the equipment rather than the athletes. To our knowledge, no study to date has focused on tennis players' perception of different mechanical and dynamic properties of rackets.

The aims of the present experiment were to assess rackets in a similar way as we did for skis in our previous study by presenting several different types of rackets to two groups of tennis players (intermediate and experts), and to examine the influence of rackets' static and dynamic properties on the "feel" and judgement of tennis players. A racket is a complex system because it is composed of several parts, frame and string, and the string tension is a key parameter that is difficult to assess in our study. Therefore, we chose to begin with a simpler system with a simple geometry and used wood-based racket 
paddles. This choice of rackets also allowed us to minimize the sweet spot issue. The sweet spot is the centre of percussion of the racket, which gives the player the impression of hitting the ball "just right" when it is aligned with the ball's trajectory. It is generally seen to correspond to the impact point that imparts maximum speed to the ball, or minimize the impulse force transmitted to the hand, thus corresponding to a vibration node. It is often the case, however, that the impact of ball and racket occurs at a different location on the racket. Our interest was mainly in the entire racket and how it is perceived as a whole rather than investigating the effect of the sweet spot. In this regard, racket paddles were ideal implements, since they do not have any preferred region to hit, although they do have a sweet spot, as any percussion device has (Haake et al., 2003; Timme \& Morrisson, 2009).

To investigate how sensitive tennis players of different standards are to changes in racket mechanical properties, we used four paddles, characterized them in the laboratory regarding their static and dynamic properties, and had them judged in the field by expert and intermediate tennis players. Finally, we correlated the subjective evaluations with the quantified measures of racket mechanical properties.

\section{Materials and methods}

\section{Materials}

We machined 12-mm thick plates of Okume wood to the geometry shown in Figure 1 to produce racket

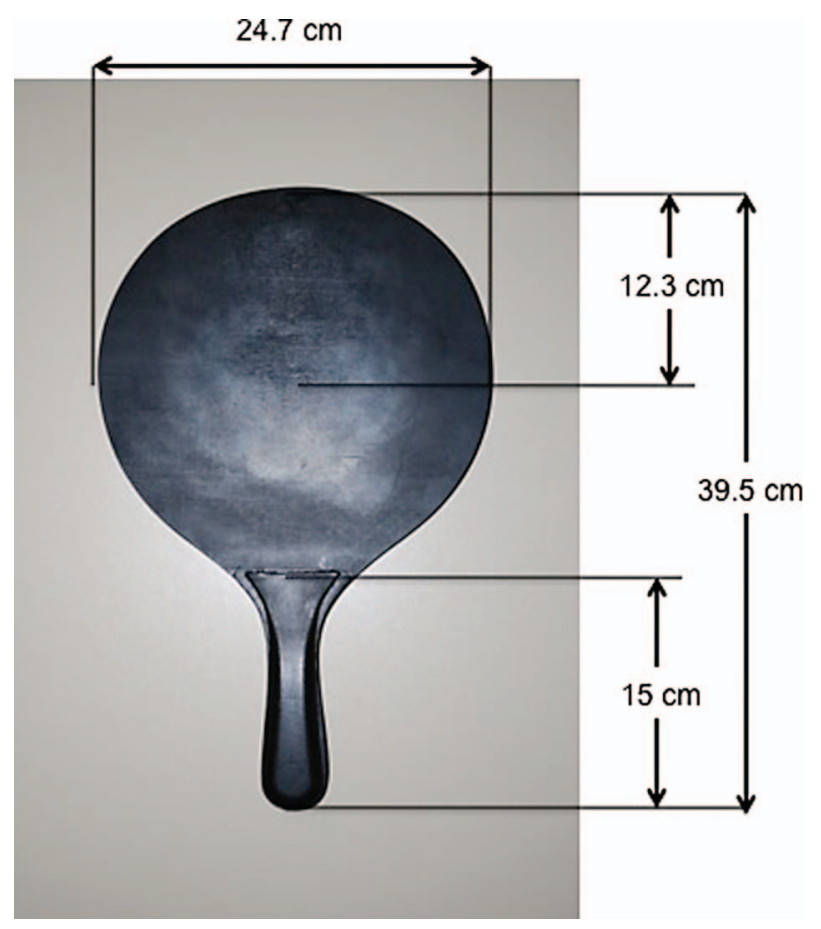

Figure 1. Picture of the type of racket used with dimensions. paddles. Before modification, all rackets weighted $319 \mathrm{~g}$ on average. Eight of the rackets were kept in their initial form to provide a statistical reference set. Three rackets were modified. The first racket, designated racket 1 or "rubber", was machined down to a thickness of $6 \mathrm{~mm}$ (to compensate for the density difference with rubber), and a layer of $1 \mathrm{~mm}$ thick rubber was glued to each side of the okume wood over the whole racket surface. The second racket, designated racket 2 or " $90^{\circ}$ carbon fibre", was an okume racket machined down to $9 \mathrm{~mm}$ thick, and covered on both sides with a three-layer carbon fibre laminate oriented at $90^{\circ}-0^{\circ}-90^{\circ}$ over the whole surface. The third, designated racket 3 or " 0 ' carbon fibre", was an okume racket machined down to $9 \mathrm{~mm}$ thick, and covered on both sides with a three-layer carbon fibre laminate oriented at $0^{\circ}-90^{\circ}-0^{\circ}$ over the whole surface. For the latter two cases, the composite was made from prepreg VTM264-T700-300g/m2-34\%wt resin from Advanced Composite Group (UK), cured according to the manufacturer's recommendations. Finally, the fourth racket, designated as racket 4 or "wood", was made of okume wood. All rackets were then painted in black. Each racket had plastic handles $15 \mathrm{~cm}$ long, glued onto them to ensure a uniform and comfortable grip during play (Figure 1). Furthermore, to ensure that each racket had the exact same mass, small pieces of lead were inserted and glued into the hollow grip handles for rackets that weighed slightly less than the heaviest one. The final mass of all the modified rackets was $474 \mathrm{~g}$. All rackets finally had the same shape, colour, and weight; only the thickness was slightly different so that it was almost impossible to distinguish them visually or by just holding them passively. The racket dimensions are given in Figure 1 and Table I.

\section{Measurement of static flexural rigidity}

The flexural rigidity of each racket was measured using a tensile tester (UTS testsysteme $\mathrm{GmbH}$ ), with a load cell of $1 \mathrm{kN}$. In a typical test, the racket handle was clamped horizontally in a grip, and the force was applied by the moving cross-head of the tester, through a spherical steel ball on the middle of the racket head that was marked on the racket (Figure 2a). A compression speed of $5 \mathrm{~mm} \cdot \mathrm{min}^{-1}$ was applied, up to a maximum of $900 \mathrm{~N}$. Each test was performed once.

First, a series of eight wooden rackets of type 4 was tested for variability in the base properties of the wood. The scatter was low, the curves linear with an average force versus displacement slope, roughly corresponding to the stiffness of the material for comparison purposes, of $23 \mathrm{~N} \cdot \mathrm{mm}^{-1}$ (standard deviation $=1 \mathrm{~N} \cdot \mathrm{mm}^{-1}$ ). Then, the four different types of rackets were tested. Figure $2 b$ presents the 
Table I. Static and dynamic measurements of the four racket types.

\begin{tabular}{|c|c|c|c|c|c|c|c|}
\hline Racket type & $\begin{array}{l}\text { Thickness } \\
(\mathrm{mm})\end{array}$ & $\begin{array}{c}\text { Flexural } \\
\text { stiffness } \\
\left(\mathrm{N} \cdot \mathrm{mm}^{-1}\right)\end{array}$ & $\begin{array}{c}\text { Torsional } \\
\text { stiffness } \\
\left(\mathrm{N} \cdot \mathrm{mm}^{-2}\right)\end{array}$ & $\begin{array}{l}\text { Damping } \\
\text { ratio }\end{array}$ & $\begin{array}{c}\text { Characteristic } \\
\text { time (s) }\end{array}$ & $\begin{array}{c}\text { First mode } \\
\text { frequency }(\mathrm{Hz})\end{array}$ & $\begin{array}{l}\text { Coefficient of } \\
\text { restitution }\end{array}$ \\
\hline 1. Rubber & 8.2 & 6 & 357 & 0.090 & 0.49 & 22.5 & 0.32 \\
\hline 2. $90^{\circ}$ carbon fibre & 11.0 & 46 & 2858 & 0.091 & 0.16 & 67.2 & 0.7 \\
\hline 3. $0^{\circ}$ carbon fibre & 11.0 & 60 & 2762 & 0.082 & 0.17 & 73.0 & 0.85 \\
\hline 4. Wood & 11.8 & 23 & 841 & 0.067 & 0.26 & 57.0 & 0.63 \\
\hline
\end{tabular}

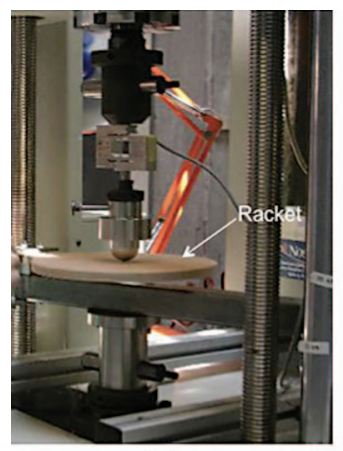

(a)

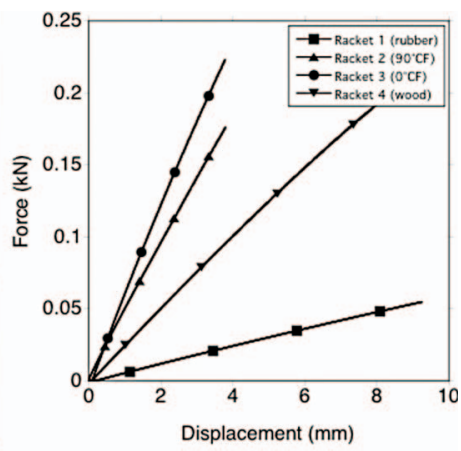

(b)

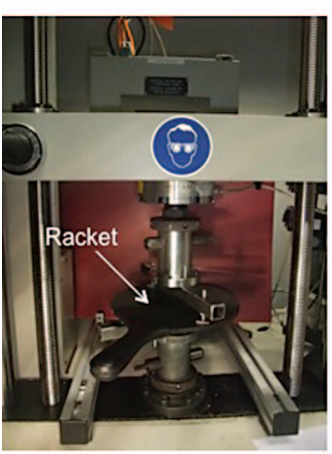

(a)

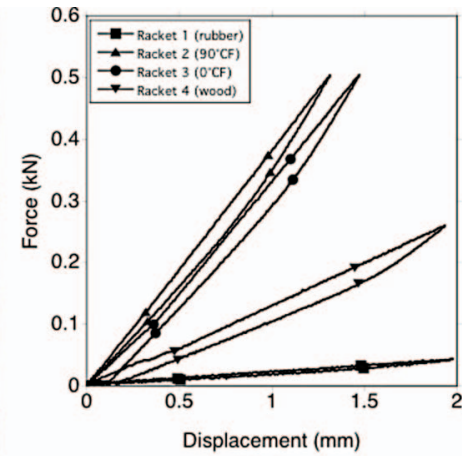

(b)

Figure 2. (a) Set-up for the bending test. (b) Typical force versus displacement curve during bending test of the four types of rackets.

force versus displacement results for the four types of rackets. In repetitive tests on the same racket, we verified that the results were consistent.

\section{Measurement of static shear rigidity}

For each racket, a measure of the rigidity in shear was performed on the tensile tester (UTS testsysteme $\mathrm{GmbH}$ ) with a load cell of $100 \mathrm{kN}$. A plate-twist setup was designed, following the ASTM D3044 norm initially designed to measure the shear modulus of plywood. In this test, shown in Figure 3(a), the racket is supported on two diagonal corners of an inscribed square of side $15 \mathrm{~cm}$, and load is applied at a constant rate of $3 \mathrm{~mm} \cdot \mathrm{min}^{-1}$ to the corners of the opposite diagonal. The stress-state induced in the plate is thus essentially shear. This method is not suitable to define the shear modulus of plates that are inhomogeneous in thickness, although we use the values to compare the apparent shear rigidity of the rackets, and not to determine the precise shear modulus of each ply. We record the displacement of the loading points and the corresponding force, and the apparent shear modulus is obtained as follows:

$$
G=\frac{3 m b^{2} K}{4 h^{3}}
$$

where $m$ is the slope of the force-displacement curve, $b$ the side of the square ( $15 \mathrm{~cm}$ in our case), $h$ the thickness of the racket as given in Table $\mathrm{I}$, and $K$ is a

Figure 3. (a) Set-up for the plate-twist test. (b) Typical force versus displacement curve during shear test of the four types of rackets.

correcting factor, a function of the ratio $r$ of the test span diagonal length, over the plate diagonal length, 0.86 in our case:

$$
K(r)=3 r^{2}-2 r-2(1-r)^{2} \ln (1-r)
$$

Figure 3(b) shows typical force-displacement curves for each case. As slight hysteresis was found between loading and unloading, the slope was taken on the loading curve. We repeated the tests twice on each racket, switching top and bottom, and the tests were reproducible with an error of $4 \%$ at worst for Racket 1.

\section{Measurement of dynamic properties}

The dynamic properties of a racket are a crucial element, because unnecessary large vibrations could cause injury to the player (Lammer, 2005) and lead to poor control of the shot. However, some vibrations may be useful to improve the feeling and control of the ball trajectory. We tested the natural damping behaviour of the four types of rackets using a Vibration Beam Testing apparatus, described in detail Fischer et al. (2006).

The racket was clamped from the handle, using a dynamometric screwdriver with a torque of $15 \mathrm{~N} \cdot \mathrm{m}$. Then, a Wilson staff squash ball was dropped vertically from a constant height of $80 \mathrm{~cm}$ through an aluminium tube, hitting the racket at the centre of the head. An accelerometer attached to the underside of the racket's head centre recorded the movement of the 


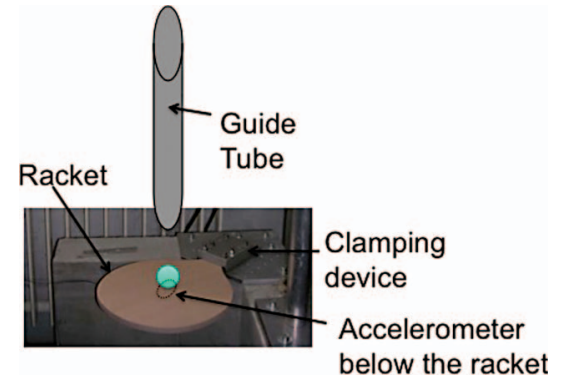

Figure 4. Schematic representationof the Vibration Beam Tester.

racket after impact. The set-up is shown in Figure 4. From the accelerometer signal, a displacement versus time curve is calculated. This curve is a periodic function, bounded in an exponentially decaying envelope (Kelly, 2007):

$$
x(t)=A \exp \left(-\xi 2 \pi f_{n} t\right) \sin \left(2 \pi f_{d} t+\phi\right)
$$

where $A$ is the maximal amplitude recorded, $\xi$ the damping ratio, $f_{d}$ the natural damped frequency, $f_{n}$ the natural frequency, with $f_{d}=f_{n} \sqrt{ }\left(1-\xi^{2}\right), t$ is time, and $\phi$ is a phase angle. From this curve, several values were measured: (i) the damping ratio $\xi$, (ii) the natural damped frequency $f_{d}$, and (iii) the characteristic time, $t_{c}=1 /\left(\begin{array}{lll}\xi & 2 \pi & f_{n}\end{array}\right)$. This time corresponds to the time it takes for the signal to decay from the maximum amplitude $A$ to $A / 2.72$. It gives an indication of the time span for the vibration to decay by a given amount.

\section{Coefficient of restitution and restituted energy}

When the ball rebounds on the racket, energy is lost. This lost energy can be related to a constant of proportionality known as the coefficient of restitution (COR). A high coefficient of restitution implies that the contact surface (the racket head) has not absorbed a large amount of the ball's energy and so the ball retains a high amount of its energy with which to bounce off the racket faster. This coefficient of restitution depends on the material and can be changed with material modifications. It also depends on the energy lost in the ball upon impact, but as we used the same balls during all tests, this fact does not affect the comparison between the rackets. We determined this coefficient of restitution for the four rackets by clamping the rackets as described earlier in the Vibration Beam Testing apparatus. A projectile was made by gluing half of a Wilson Staff Squash ball to the end of an aluminium cylinder, resulting in a mass of $174 \mathrm{~g}$. A laser was positioned through the aluminium tube, and measured the velocity of the impactor both before and after impacting the racket when it was dropped from a height of $5 \mathrm{~cm} . v_{f}$ is the velocity after impact, and $v_{o}$ the velocity before impact. The coefficient of restitution was then calculated from the following equation: $\mathrm{COR}=v_{f} / v_{o}$.

\section{Assessment of perceived characteristics of the rackets}

Participants. The participants were either competing at a national level (expert players) or at a regional level (intermediate players). They were assigned to one of the groups depending on their rankings. Seven expert (mean age 24.0 years, $s=6.0$ ) and eight intermediate (mean age 26.6 years, $s=6.7$ ) male tennis players took part in the study. There were two left-handers in each group.

Procedure. The experiment took place on an outdoor tennis court at the Lausanne University sports centre. Once participants received all instructions, they began with a short warm-up that involved playing tennis against a tennis wall with a training racket paddle for $5 \mathrm{~min}$. This training racket paddle was the same as the ones used for the test (same size, weight, shape, and colour), although it was in its original form (i.e. not modified with any extra material) and was not used in the actual test. New "Wilson" tennis balls were used for the field tests.

Then, the participants played with the first test racket for $1 \mathrm{~min}$ and straight afterwards they played with the second test racket for $1 \mathrm{~min}$. The players were asked to hit forehands and backhands only (no volleys or serves). The rackets were tested in pairs with $1 \mathrm{~min}$ play per racket. The time between two rackets of a same pair was very short (about $30 \mathrm{~s}$ ), just enough to exchange the rackets. Four rackets in total were used for the field testing.

Each player thus compared two of the four rackets at a time in all 16 different combinations of racket pairings ( 1 vs. 1,1 vs. 2,1 vs. 3,1 vs. 4,2 vs. 1,2 vs. 2 , etc.), the order of which was randomized. Note that " 1 vs. 1 " means that the same racket was used twice for a given comparison. This was done to determine whether players would be able to detect that they were using the same rackets or not. After playing with both rackets of one pair, they completed a short questionnaire asking them to compare one racket with the other. The questions were as follows: (Q1) Which racket needed more force? (Q2) Which racket had better control? (Q3) Which racket had more vibration? (Q4) Which racket did you prefer? They had to answer " 1 " or " 2 " to each question. The first three questions were chosen based on the quantified measures of the rackets' properties. Thus, Q1, Q2, and Q3 are expected to relate to flexural properties (or stiffness), damping, and restituted energy, respectively.

After testing the 16 combinations, we asked the tennis players to perform a discrimination task. 
Again they had to compare rackets two by two and tested each one during $1 \mathrm{~min}$ of play, but had to determine whether the two rackets were the same or different. Eight combinations were tested: four identical (e.g. 2 vs. 2 ) and four different (e.g. 2 vs. 3) pairs.

Overall, the whole procedure lasted $1 \mathrm{~h}$.

Data analysis. We first determined the correlations between the various mechanical properties of the four rackets (Table II), using Pearson's correlation coefficient $(r)$. Our main aim was to determine how tennis players of two different standards would judge four different types of rackets. More specifically, we were interested in how differently these groups would judge the rackets. Therefore, we used a method of analysis that would help us visualize these differences clearly. Thus, to reveal relationships between the four different rackets in the subjective data, we carried out a multi-dimensional scaling. Multi-dimensional scaling allows one to visualize how near points are to each other for many kinds of distance or dissimilarity metrics and can produce a representation of the data in a small number of dimensions. In the present case, three dimensions were necessary, one for each of the first three questions.

We used non-metric multi-dimensional scaling. This type of multi-dimensional scaling assumes only an ordinal relationship between the data and the derived inter-stimulus distance (Kruskal \& Wish, 1978). Accordingly, to treat the data, we can use any method that preserves the ordinal relations among the data. Thus we made score matrices from participants' responses according to the following method. If a player answered that he had better control with racket 2 than with racket 1 , then 1 point was given to racket 2 . If players answered that both rackets were the same, then 1 point was given to each racket.

To analyse the data by multi-dimensional scaling, dissimilarity matrices representing inter-stimulus psychological distances are needed (Kruskal \& Wish,
1978). We computed profile distances to obtain the dissimilarity matrices, using the XLSTAT program.

A multi-dimensional scaling algorithm starts with a matrix of item-item similarities and then assigns a location to each item in $\mathrm{N}$-dimensional space, where $N$ is specified a priori. For sufficiently small $N$, the resulting locations may be displayed in a graph or 3D visualization. Multi-dimensional scaling uses a function minimization algorithm that evaluates different configurations with the goal of maximizing the goodness-of-fit. For any given configuration, a monotone regression of distance upon dissimilarity is performed. The residual variance, suitably normalized, is used as the quantitative measure. This is called the "Stress". Thus for any given configuration, the Stress measures how well that configuration matches the data (for more details, see Kruskal, 1964).

Correlations were also analysed between the judged properties (via questions Q1, Q2, and Q3) and the corresponding quantified values [flexural properties (or stiffness), damping, and restituted energy], using Pearson's correlation coefficient $(r)$.

\section{Results}

Mechanical properties of the rackets and their correlations

Flexural rigidity values obtained from the slope of the load-displacement curves in the flexion measurements are reported in Table I. We observed meaningful differences between the four types of rackets and, as expected, the use of carbon-fibre laminate increased the rigidity of the racket.

Apparent shear modulus is also reported in Table I. The outcome is similar, although the $90^{\circ}$ racket showed a slightly higher rigidity in shear. Form the vibration experiments, we obtained the damping ratio $\xi$, the natural damped frequency $f_{d}$, and the characteristic time, $t_{c}=1 /\left(\xi 2 \pi f_{n}\right)$. These three values are reported in Table I for the four types of racket. The coefficient of restitution is also reported in Table I.

Table II. Results of the statistical correlations between the mechanical parameters using Pearson's correlation coefficient ( $r$ ).

\begin{tabular}{lccccrr}
\hline Variable & $\begin{array}{c}\text { Flexural } \\
\text { stiffness }\end{array}$ & $\begin{array}{c}\text { Torsional } \\
\text { stiffness }\end{array}$ & $\begin{array}{c}\text { Damping } \\
\text { ratio }\end{array}$ & $\begin{array}{c}\text { Characteristic } \\
\text { time }\end{array}$ & $\begin{array}{c}\text { First mode } \\
\text { frequency }\end{array}$ & $\begin{array}{c}\text { Coefficient of } \\
\text { restitution }\end{array}$ \\
\hline Flexural stiffness & 1.00 & $\mathbf{0 . 9 5}$ & 0.06 & -0.90 & 0.92 & 0.95 \\
Torsional stiffness & $\mathbf{0 . 9 5}$ & 1.00 & 0.29 & -0.88 & 0.85 \\
Damping ratio & 0.06 & 0.29 & 1.00 & 18 & -0.24 & -0.25 \\
Characteristic time & -0.90 & -0.88 & 0.18 & $-\mathbf{0 . 9 9}$ & $\mathbf{- 0 . 9 5}$ \\
First mode frequency & 0.92 & 0.86 & -0.24 & $-\mathbf{0 . 9 9}$ & $\mathbf{0 . 9 0}$ & $\mathbf{0 . 9 8}$ \\
Coefficient of restitution & 0.95 & 0.85 & -0.25 & $\mathbf{0 . 9 5}$ & 1.00 \\
\hline
\end{tabular}

Note: Values in bold correspond to statistically significant results $(P<0.05)$. 
The correlation analysis for the mechanical response of the rackets revealed four significant correlations (Table II). Flexural stiffness was highly correlated with torsional stiffness $(r=0.95$, $P<0.05)$. Characteristic time was negatively correlated with both first mode frequency $(r=-0.99$, $P<0.05)$ and the coefficient of restitution ( $r=-0.95, \quad P<0.05)$. Furthermore, first mode frequency was strongly correlated with the coefficient of restitution $(r=0.98, P<0.05)$. The damping ratio did not correlate with any of the other properties.

\section{Evaluation of the subjective ratings}

Figures $5 \mathrm{a}$ and $5 \mathrm{~b}$ show the relationships between the four different rackets according to three axes, which correspond to the first three questions of the questionnaire about force, control, and vibration. The axes for force and vibration were switched to positive for the sake of clarity. It is important to note that high scores on the force and vibration axes are considered bad results for the racket, since a racket requiring a strong force and which vibrates a lot is a priori not a good racket. Interestingly, our results show that expert (Figure 5a) and intermediate (Figure 5b) tennis players have very similar racket preferences. The carbon fibre rackets were largely preferred over the wood and rubber rackets (see Figures $5 \mathrm{a}$ and $\mathrm{b}$ ). The graphs clearly show that the carbon fibre rackets (numbers 2 and 3) are rated as good rackets because they have low force and low vibration but high control. In contrast, the rubber racket is rated the worst racket of the four with high ratings on the force and vibration axes and low control. The wood racket lies in between.
The two carbon fibre rackets were judged very similarly and this was also reflected in the subjective reports and in the discrimination task. Question 4 on the questionnaire was related to the players' racket preference. The majority of players $(79.9 \%)$ reported a preference for one of the carbon fibre rackets. Only $13.3 \%$ preferred the wooden racket and only one player $(6.6 \%)$ liked the rubber racket best. More specifically, all expert players preferred the carbon fibre rackets $(71.5 \%$ preferred racket 3 and $28.5 \%$ preferred racket 2), whereas the intermediate players' choices were more variable $37.5 \%$ preferred racket 3, 25\% preferred racket 2, 25\% preferred racket 4 , and $12.5 \%$ preferred racket 1 ). In the discrimination task, expert players showed $64.5 \%$ correct responses whereas intermediate players scored, surprisingly, $75 \%$ correct.

\section{Correlations between subjective ratings and mechanical properties}

One of our main goals was to investigate the potential correlation between the tennis players' judgement and the physical properties of the four types of rackets. We therefore correlated the data reported in Table I with the ratings of the participants using Pearson's correlation coefficient $(r)$. The results of these correlations are shown in Table III. Please note that the correlations were performed on the raw data, such that force and vibration have negative values. Hence, a positive correlation between force or vibration and one of the mechanical properties means that they are in fact inversely correlated (for example, if force and stiffness are positively correlated in Table III, it means that the stiffer the racket, the less force is required, hence the racket seems to perform better). Some differences were observed

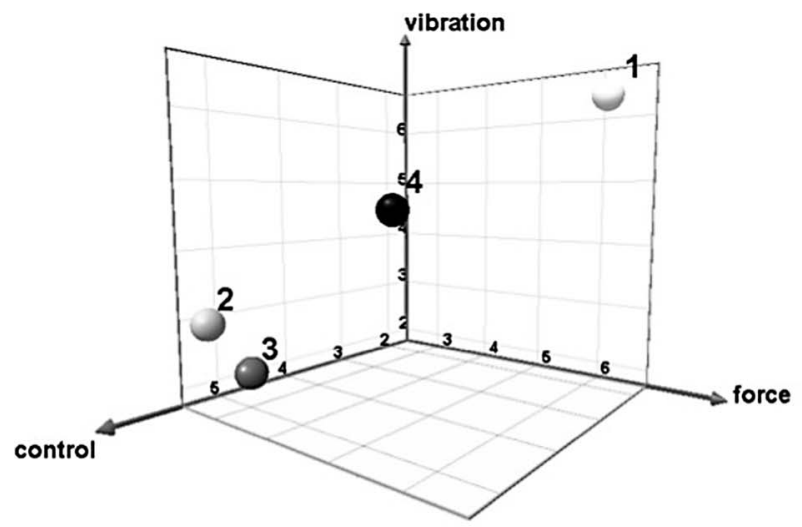

(a)

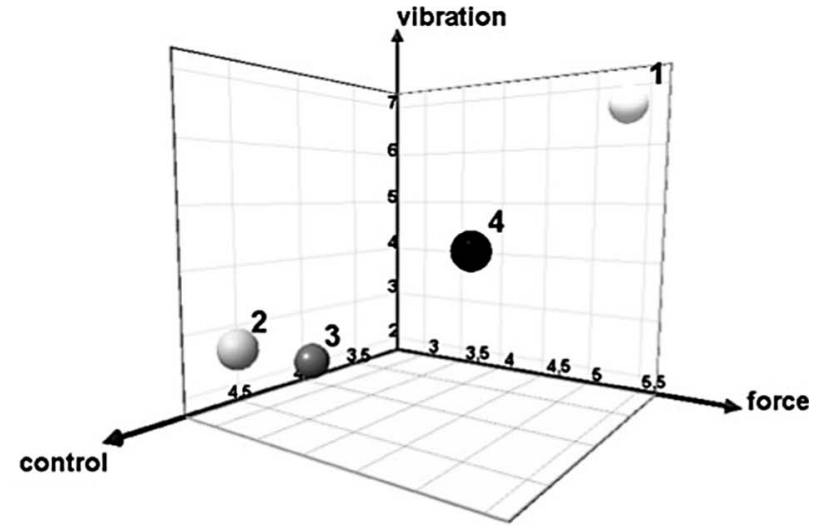

(b)

Figure 5. (a) Racket preference for the expert players. Racket 1 is the rubber racket; racket 2 is the $90^{\circ}$ carbon fibre racket; racket 3 is the $0^{\circ}$ carbon fibre racket; and racket 4 is the wood racket. The three axes represent the first three questions of our questionnaire: $\mathrm{X}$-axis represents force (high force is bad), Y-axis represents control (high control is good), and Z-axis represents vibration (high vibration is bad). (b) Racket preference for the intermediate players. Numbers and axes are the same as for (a). 
Table III. Results of the statistical correlations between subjective ratings and objective data using Pearson's correlation coefficient $(r)$.

\begin{tabular}{lccccc}
\hline Variable & Stiffness & Damping & Characteristic time & First mode frequency & Coefficient of restitution \\
\hline Force, experts & 0.93 & 0.02 & $-\mathbf{0 . 9 8}$ & $\mathbf{0 . 9 6}$ & 0.92 \\
Control, experts & 0.91 & -0.09 & $-\mathbf{1 . 0 0}$ & $\mathbf{0 . 9 8}$ & 0.94 \\
Vibration, experts & $\mathbf{0 . 9 9}$ & -0.00 & 0.95 & $\mathbf{0 . 9 6}$ & 0.93 \\
Force, intermediates & $\mathbf{0 . 9 8}$ & 0.14 & -0.93 & 0.87 & 0.92 \\
Control, intermediates & 0.76 & 0.01 & -0.93 & $\mathbf{0 . 9 9}$ & 0.77 \\
Vibration, intermediates & $\mathbf{0 . 9 7}$ & -0.10 & $-\mathbf{0 . 9 8}$ & $\mathbf{0 . 9 8}$ \\
\hline
\end{tabular}

Note: Values in bold correspond to statistically significant results $(P<0.05)$.

between expert and intermediate players. While rigidity only correlated with vibration $(r=0.99$, $P<0.05)$ in expert players, it correlated with both vibration $(r=0.97, P<0.05)$ and force $(r=0.98$, $P<0.05)$ in intermediate players. Surprisingly, damping did not correlate significantly with any ratings of the players. Characteristic time clearly segregated the two groups, since it correlated inversely with force $(r=-0.98, P<0.05)$ and control $(r=-1.00, P<0.05)$ but not vibration in experts, while the opposite pattern was observed for the less skilled players (characteristic time was inversely correlated with vibration: $r=-0.98$, $P<0.05)$. First mode frequency was highly correlated with all ratings of the expert players (force: $r=0.96, P<0.05$; control: $r=0.98, P<0.05$; vibration: $r=0.96, P<0.05$ ), whereas only vibration $(r=0.99, P<0.05)$ was correlated with first mode frequency of intermediate players' ratings. Finally, the coefficient of restitution correlated with vibration in both expert $(r=0.97, P<0.05)$ and intermediate $(r=0.98, P<0.05)$ ratings.

\section{Discussion}

In the present study, we were interested in tennis players' judgement of rackets. We presented intermediate and expert players with four different types of racket paddles and examined the influence of these rackets' static and dynamic properties on the "feel" and judgement of the players.

First, the correlations between the rackets' mechanical properties revealed that the results were in accordance with physical expectations. For instance, characteristic time is inversely proportional to first mode frequency, which is what was observed with the significantly negative correlation between the two. The ranking in stiffness is quite close to that in shear modulus, except for the carbon rackets, as would be expected, since their lay-up was different.

Regarding subjective ratings, overall the $0^{\circ}$ carbon fibre racket was the most preferred, followed very closely by the $90^{\circ}$ carbon fibre, the wood, and finally the $1-\mathrm{mm}$ rubber racket. This result translates into a preference for a direct force transfer from the racket to the athlete, which is characteristic of the $0^{\circ}$ carbon fibre racket. Although we expected differences between expert and intermediate players, none were found in this general classification. This could be potentially explained by the fact that all players are tennis players, and thus new to the paddle rackets they were using. This may have placed all players on a more equal ground, in terms of feel.

Differences between expert and intermediate players arose from the correlations between subjective ratings and objective data. Characteristic time was clearly the property that discriminated the two groups most. While characteristic time was inversely correlated with required force and control in experts, it was inversely correlated with vibration in the less skilled players. In that sense, intermediate players were closer to reality in their judgement than experts, since characteristic time indeed negatively correlated with first mode frequency (a measure of vibration). This is in agreement with previous research on the correlation between mechanical properties and subjective appreciation of skis, where intermediate skiers were more sensitive to damping differences between skis than experts (Fischer et al., 2007).

Experts were more sensitive to the first mode frequency than intermediates. Differences in damping between the rackets, however, were not perceived by any of the players. Interestingly, characteristic time, first mode frequency, and damping are all parameters that are linked to vibration. We had expected tennis players to be able to perceive damping differences between the rackets, which was not the case. Results show that they were more sensitive to the other two parameters, namely, characteristic time and first mode frequency, suggesting that in terms of perception, the vibration duration over-rates the actual damping ratio. This again would mean that quickly vanishing high frequencies should be preferred over highly damping materials in racket design.

Finally, stiffness and the coefficient of restitution both mainly correlated with vibration in both groups. According to the measurement results, the carbon fibre rackets were considerably stiffer than the plain wood racket, which in turn was stiffer than the 
rubber-covered racket. Due to the higher amount of carbon fibres oriented along the racket axis (i.e. in $0^{\circ}$ direction), the carbon fibre racket $0^{\circ}-90^{\circ}-0^{\circ}$ was stiffer than the $90^{\circ}-0^{\circ}-90^{\circ}$ laminate, resulting in a more direct force transfer compared with the $90^{\circ}$ racket. In a $90^{\circ}$ racket, the fibres are arranged perpendicular to the pulling force and have less stiffness when the racket is bent. When the force from the ball on the racket is removed, the vibration frequency of the stiffer racket will be higher, and thus dissipate the energy faster. This implies that a stiffer racket is more comfortable for an athlete to use because it will vibrate for less time than a more flexible racket. Furthermore, a stiffer racket has less contact time with the ball than a more flexible racket because a stiffer racket deforms less upon contact. Therefore, the racket frame absorbs less of the ball's energy and so there is less energy available to cause vibrations.

The $0^{\circ}$ carbon fibre racket had the highest coefficient of restitution, followed by the $90^{\circ}$ carbon fibre, the wood, and finally the 1-mm rubber racket. Since the athletes' preferences (Question 4) were exactly in this order, it can then be presumed that as the coefficient of restitution increases, the racket becomes more favourable to the greatest number of tennis players. This is in line with Haake et al. (2003), who suggested that tennis players prefer tennis balls of high stiffness, since they generally imply a high coefficient of restitution and are therefore perceived to fly faster off the racket.

A final interesting result came from the discrimination task in which intermediate players performed better than experts. Thus, training and expertise does not necessarily improve sensitivity to differences in mechanical and dynamical properties of rackets; rather, sensitivity seems to develop towards other parameters. As revealed by our correlations between subjective judgements and objective data, more experienced players were influenced by characteristic time and first mode frequency, while intermediate players were influenced more by stiffness. Alternatively, it could be argued that expert players adapt themselves faster to new equipment than intermediate players, which could partly explain their poor performance in the discrimination task. However, it is important to note that we did not use actual tennis rackets but rather tennis racket paddles without strings. Thus, this study should be considered as a starting point for future studies in the field and further investigations with actual tennis rackets are required before drawing premature conclusions on real tennis and tennis players. We believe that the present results provide good insight for studies with real rackets and that the latter will be crucial for future racket designs and developments.

\section{Acknowledgements}

This project was funded by the Sport and Rehabilitation program at EPFL. M. S. Lavanchy and A. Sigg are warmly acknowledged for support with the mechanical testing experiments.

\section{References}

Carello, C., Thuot, S., Anderson, K. L., \& Turvey, M. T. (1999). Perceiving the sweet spot. Perception, 28, 307-320.

Cross, R. (1998). The sweet spot of a tennis racquet. Sports Engineering, 1, 63-78.

Fischer, C., Braun, S. A., Demarco, F., Bouban, P.-E., Michaud, V., Plummer, C. J. G. et al. (2006). Dynamic properties of sandwich structures with integrated shear thickening fluids. Smart Materials and Structures, 15, 1467-1475.

Fischer, C., Overney, L. S., Fauve, M., Blanke, O., Rhyner, H., Herzog, M. H. et al. (2007). What static and dynamic properties should slalom skis possess? Judgements by advanced and expert skiers. Fournal of Sports Sciences, 25, 1567-1576.

Haake, S. J., Carré, M. J., \& Goodwill, S. R. (2003). The dynamic impact characteristics of tennis balls with tennis rackets. Fournal of Sports Sciences, 21, 839-850.

Kelly, S. G. (2007). Advanced vibration analysis. Boca Raton, FL: CRC Press/Taylor \& Francis.

Kruskal, J. B. (1964). Nonmetric multidimensional scaling: A numerical method. Psychometrika, 29, 115-129.

Kruskal, J. B., \& Wish, M. (1978). Multidimensional scaling. Newbury Park, CA: Sage.

Lammer, H. (2005). Head's tennis racket IS 18 chipsystem and ski IC 300 chipsystem - smart materials for serial applications. Proceedings of the 26th SAMPE Europe Conference. Paris.

Roberts, J. R., Jones, R., Harwood, C., Mitchell, S., \& Rothberg, S. J. (2001). Human perceptions of sports equipment under playing conditions. Fournal of Sports Sciences, 19, 485-497.

Roberts, J. R., Jones, R., Mansfield, N. J., \& Rothberg, S. J. (2005a). Evaluation of vibrotactile sensations in the "feel" of a golf shot. Fournal of Sound and Vibration, 285, 303-319.

Roberts, J. R., Jones, R., Mansfield, N. J., \& Rothberg, S. J. (2005b). Evaluation of impact sound on the "feel" of a golf shot. Fournal of Sound and Vibration, 287, 651-666.

Stroede, C. L., Noble, L., \& Walker, H. S. (1999). The effect of tennis racket string vibration dampers on racket handle vibrations and discomfort following impacts. Fournal of Sports Sciences, 17, 379-385.

Timme, N., \& Morrisson, A. (2009). The mode shapes of a tennis racket and the effects of vibration dampers on those mode shapes. Fournal of the Acoustical Society of America, 125, 36503656. 